\title{
Las Competencias Docentes Genéricas en los Grados de Educación. Visión del Profesorado Universitario*
}

\author{
Generic Teaching Competences in Education Degree Programs: \\ The Vision of the University Faculty
}

\section{Antonia Ramírez-García ${ }^{a}$, Natalia González-Fernández ${ }^{b}$, Irina Salcines-Talledoc}

\author{
Universidad de Córdoba \\ Correo electrónico: a.ramirez@uco.es \\ Universidad de Cantabria, Telf.: (34) 942201270 \\ Correo electrónico: gonzalen@unican.es \\ Universidad de Cantabria, Telf.: (34) 942201883 \\ Correo electrónico: salcinesi@unican.es
}

\begin{abstract}
RESUMEN
El enfoque competencial en Educación Superior, promovido a partir del proceso de convergencia europea, ha adquirido una gran importancia y relevancia en los procesos formativos actuales. Este trabajo trata de identificar las competencias genéricas que se están implementando en las aulas universitarias y su grado de desarrollo, analizando las diferencias existentes en función de las características de los docentes. Para dar respuesta al objetivo planteado, 351 docentes universitarios españoles han cumplimentado un cuestionario como técnica cuantitativa para la recogida de datos. Los principales resultados de la investigación reflejan que se promueve el desarrollo de la mayor parte de las competencias genéricas en las aulas, siendo la relacionada con la gestión y los vínculos con el contexto social las menos desarrolladas. Cabe destacar la formación permanente del profesorado como factor determinante en la promoción de dichas competencias.
\end{abstract}

Palabras claves: enfoque competencial, futuros docentes, competencias docente genéricas, profesorado universitario

\begin{abstract}
The competence approach in higher education, promoted through the process of European convergence, has acquired great importance and relevance in current teacher training processes. This work aims to identify the generic teaching competences that are being implemented in university classrooms and their degree of development, analyzing the existing differences according to the characteristics of university professors. To achieve the proposed objective, 351 Spanish university professors were given a questionnaire as a quantitative technique for collecting data. The main results of the study reveal that the development of most generic teaching competences was being promoted in the classroom. However, those competences related to management and the social context were the least developed. It is worth highlighting the continuing education of university professors was a determining factor in the promotion of said competences.
\end{abstract}

Keywords: competence approach, future teachers, generic teaching competences, university faculty.

Programa Estatal de Investigación, Desarrollo e Innovación Orientada a los Retos de la Sociedad (I+D+i), en el marco del Plan Estatal de Investigación Científica y Técnica y de Innovación 2013-2016. Referencia: EDU 2013-42024-R. 


\section{INTRODUCCIÓN}

La Declaración de Bolonia (1999) posibilitó un cambio sustancial en los sistemas educativos nacionales de los diferentes estados miembros con el fin de una necesaria convergencia supranacional, para adaptarse al proceso del Espacio Europeo de Educación Superior (Ángulo, 2009; Lavega, 2008). En este sentido, los distintos países generaron una abundante legislación al respecto que permitió el nacimiento de los planes de estudio de las nuevas titulaciones de grado y postgrado.

Los planes de estudio de las diferentes titulaciones universitarias se han diseñado en torno a un modelo de formación basado en competencias, aspecto vinculado no solo a los elementos curriculares, sino también a la profesionalización de los estudios. Esta característica los sitúa, de acuerdo con Cascante (2004), en el marco de los discursos técnicos de la formación profesional, que organizan los planes de estudio a partir de los perfiles profesionales y configuran un conjunto de asignaturas para que los estudiantes adquieran las competencias necesarias en el desempeño de dichos perfiles profesionales que, como afirma Tejada (2009), se concretan en una determinada familia profesional.

Al mismo tiempo, esta profesionalización exige nuevas formas de enseñar, nuevas formas de aprender y cambios en la planificación y estructuración de la educación. Este hecho ubica a los estudios universitarios en la esfera de acción del discurso práctico, caracterizado por un proceso de reflexión en la acción y sobre la acción, en permanente contacto con la práctica y sobre la base de una formación que se integra en la investigación práctica y en el trabajo en seminarios, entre otros.

Este nuevo modelo formativo ha ido generando una nueva cultura de la formación, una propuesta formativa centrada en la adquisición de competencias para el ejercicio de la profesión (Lozano et al., 2017), es decir, lo que para Lasnier (2000) supone un saber hacer complejo resultado de la integración, movilización y adecuación de capacidades, habilidades y conocimientos utilizados eficazmente en situaciones que tengan un carácter común.

Se trata, según Le Boterf (2001), de formar a un profesional que sepa gestionar y manejar una situación compleja, actuar y reaccionar con pertinencia, combinar los recursos y movilizarlos en un contexto, comprender y transferir, así como aprender a aprender.

Tal y como refleja el Proyecto Tuning (González \& Wagenaar, 2003), el término competencia representa una combinación de atributos (con respecto al conocimiento y sus aplicaciones, aptitudes, destrezas y responsabilidades) que describen el nivel o grado de suficiencia con que una persona es capaz de desempeñarlos. Este concepto se encuentra estrechamente relacionado con otros términos con significados similares como capacidad, atributo, habilidad y destreza.

Por su parte, los responsables del Proyecto DeSeCo (Definition and Selection of Competencies) también formularon la siguiente definición respecto al concepto de competencia:

Vista desde fuera una competencia puede ser definida como la habilidad que permite superar las demandas sociales o individuales, desarrollar una actividad o una tarea. Vista desde dentro, cada competencia es construida como una combinación de habilidades prácticas, conocimientos (incluyendo conocimiento tácito), motivación, valores éticos, actitudes, emociones y otros componentes sociales y de comportamiento 
que pueden movilizarse conjuntamente para que la acción realizada en una situación determinada pueda ser eficaz. (OCDE-DeSeCo, 2002, p. 8).

El análisis de diferentes definiciones sobre el término competencia (Barnett, 2001; Cano, 2005, 2007, 2012; Delamare \& Winterton, 2005; Román, 2007; Zabalza, 2002) nos lleva a afirmar el carácter polisémico del mismo, destacando entre ellas la definición dada por Zabala y Arnau (2008) sobre el citado término y de la que partimos para concluir que una competencia:

Consistirá en la intervención eficaz en los diferentes ámbitos de la vida mediante acciones en las que se movilizan, al mismo tiempo y de manera interrelacionada, componentes actitudinales, procedimentales y conceptuales, todo ello enmarcado en un proceso que exige el seguimiento de una serie fases de diferente complejidad en el menor tiempo posible. Estas fases serían las siguientes: análisis del contexto, planificación de la actuación, ejecución de la acción y revisión de los resultados de la acción. (p. 45).

Para estos autores, el término "competencia" no indica tanto lo que uno posee como el modo en que uno actúa en situaciones concretas para realizar las tareas de forma excelente. No se puede afirmar que una persona es capaz de demostrar cierta competencia hasta el momento en que aplica esos conocimientos, habilidades y actitudes en la situación adecuada, resolviéndola de forma eficaz. Por este motivo, las competencias tienen implícito el elemento contextual, referido al momento de aplicar estos saberes a las tareas que la persona debe desempeñar. De este modo, puesto que cada situación es única y diferente de las demás, por muchos elementos que compartan, es posible que una persona demuestre unas competencias en unas situaciones y no en otras. Por su parte, Perrenoud (2004) también advierte la importancia del contexto, ya que para él una competencia es un conjunto de conocimientos, destrezas y actitudes que ha de ser capaz de movilizar una persona de forma integrada, para actuar eficazmente ante las demandas de un contexto determinado.

Diferentes teorías han analizado la cuestión contextual, así podemos destacar la teoría ecológica de Bronfenbrenner (1987), inserta en el marco de las teorías dialécticas contextuales, que explican el cambio de conducta de los sujetos mediante la influencia del entorno del contexto. En sus trabajos, Bronfenbrenner (1987) mantiene que el desarrollo de los seres humanos queda condicionado por su situación familiar, laboral y socioeconómica, al tiempo que por las interacciones que se producen en una serie de sistemas, ya tradicionales, que se superponen -microsistema, mesosistema, exosistema, macrosistema-contextos en los que el sujeto aprende y se desenvuelve.

En este sentido, la perspectiva competencial vuelve sus ojos al contexto, destacando que las competencias básicas se adquieren y aplican en diferentes contextos: individual o personal, familiar, escolar o educativo, en la comunidad -público o profesional-. En esta mirada hacia los contextos de desarrollo humano hemos detectado la carencia de un nuevo contexto, que si bien pudiera estar implícito en algunos de los ya mencionados debido a su carácter transversal, merece un tratamiento especial, nos referimos al contexto virtual, al ciberespacio, un nuevo entorno de aprendizaje y de aplicación de competencias vinculado a las tecnologías de la información y la comunicación, este contexto, al igual que los que mencionaba Bronfenbrenner (1987), altera, modifica el desarrollo de la persona. 
El cambio metodológico que supone la adopción de un modelo formativo basado en competencias conlleva un análisis y planificación de los contextos en los que se van a aprender y aplicar dichas competencias. De este modo, para Gros y Bernat (2008, p. 14) "el diseño de los contextos de aprendizaje se convierte en una de las tareas básicas para el profesor, por lo que el rol de este cambia de forma muy notable”. Desde esta posición, es necesario prestar atención al contexto -formal, no formal, informal-.

Las competencias se movilizan, pues, en los diferentes escenarios que configuran el contexto en el que vivimos. En este sentido, Monereo y Pozo (2007) señalan que las competencias se ponen en acción en contextos problemáticos que se definen por su autenticidad, es decir, que se definen como reales (fieles a las condiciones de la vida real) y relevantes (vinculados al quehacer vital y supervivencia). Por su parte, Coll (2007, p. 36) no solo "alude al contexto de aplicación de las competencias básicas, sino que también hace referencia al contexto en el que se adquieren". De este modo, podemos afirmar que las competencias no pueden desligarse de ambos contextos, el de aprendizaje y el de aplicación.

Al mismo tiempo hay que considerar que las competencias, por sí mismas, no pueden desarrollarse. Estas requieren el establecimiento de una serie de descriptores y aspectos distintivos que las pormenorizan y que aluden a los distintos procesos implícitos en las mismas (Bloom, 1956; Estévez, 2002; Krathwohl, Bloom \& Masia, 1964; RodríguezDiéguez, 1980), procesos cognitivos, afectivos, sociales y funcionales o estratégicos, a los que podríamos añadir los procesos motrices (Ramírez-García, 2013).

Se trata, pues, de poner en práctica una formación que integre el espectro profesional y el ámbito cultural y social, contemplando en su puesta en marcha una serie de dimensiones que Rué (2004) reseña como dominio de conocimientos propios de su ámbito formativo, desarrollo del conocimiento científico, habilidades de resolución de problemas, capacidad de trabajo en grupo, orientación ética del trabajo profesional y formación continua.

Estas dimensiones deben quedar reflejadas en las competencias que el alumnado universitario debe adquirir y desarrollar dando, según Ramos (2005), una mayor importancia a la observación y a la práctica educativa de campo, estableciendo una relación estrecha entre el aprender a conocer y el aprender a hacer.

Por ello, Perrenoud (2004) apunta una serie de aspectos sobre los que debe bascular la formación en competencias del alumnado universitario, tanto por parte del profesorado, que ha de modificar su forma de enseñar (Sierra, Méndez-Jiménez \& Mañana-Rodríguez, 2013), como por él mismo, que ha de cambiar su forma de aprender. Estos aspectos son los siguientes:

a) Organización y dinamización de situaciones de aprendizaje.

b) Gestión de los progresos en el aprendizaje.

c) Implicación de los estudiantes en su aprendizaje y esfuerzo.

d) Trabajo en equipo y formación de los estudiantes en el trabajo en grupo.

e) Afrontamiento los deberes y dilemas éticos de la profesión.

f) Gestión de su propia formación continua.

En esta dirección, la necesidad de llevar a cabo una formación en competencias en la universidad supondrá, entre otras (González \& Wagenaar, 2003):

a) Una mayor transparencia de los perfiles profesionales en los programas a estudio y énfasis en los resultados de aprendizaje. 
b) Cambiar a un enfoque educativo más orientado a quien aprende.

c) Respuestas a las demandas crecientes de una sociedad de aprendizaje permanente, lo que requiere mayor flexibilidad.

d) Necesidad de niveles superiores de empleo y ciudadanía.

e) Mejora de la dimensión Europea de la Educación Superior.

f) Necesidad de un lenguaje compartido para consulta entre todos los implicados.

La adquisición de las competencias por parte de los futuros docentes, como ya hemos apuntado, exige una transformación en la preparación del profesorado y del alumnado universitario, los rasgos principales del modelo educativo hacia el que nos dirigimos y que lo convierten en un modelo más eficaz para los nuevos resultados esperados del aprendizaje son, de acuerdo con Fernández-March (2005), los siguientes:

1. Modelo educativo centrado en el aprendizaje que exige el giro de la universidad del enseñar a la universidad del aprender y, sobre todo, del aprender a aprender.

2. Modelo educativo centrado en el aprendizaje autogestionado por el alumno y tutorizado por el profesor. Aprender cómo seguir aprendiendo a lo largo de la vida.

3. Modelo educativo que enfoca el proceso de Aprendizaje-Enseñanza como trabajo cooperativo entre estudiantes y profesor. El trabajo cooperativo exige interdependencia, simultaneidad y responsabilidad personal.

4. Modelo educativo centrado en los procesos y resultados del aprendizaje. Frente a la importancia de las entradas (input) cobran prioridad las salidas (output). Las competencias genéricas y específicas, académicas y profesionales del perfil del egresado, son los resultados de aprendizaje que se han de lograr en el proceso de aprendizaje-enseñanza.

5. Modelo educativo que exige una nueva concepción de las tareas de aprendizaje, enseñanza y evaluación.

6. Modelo educativo que usa la evaluación estratégicamente: formativa-continuada y final-certificativa.

7. Un Modelo educativo que mide el trabajo del estudiante en créditos ECTS.

Este modelo educativo competencial, si se analiza desde la óptica de los grandes enfoques de diseño y planificación curricular -modelo tecnológico y modelo procesual (Clemente, 2010)-, ofrece posicionamientos diferenciados en torno a las competencias (Martín-Domínguez \& Lavega, 2013). En cualquier caso, exige el desarrollo de un perfil profesional, de unos roles y unas actividades diferentes a los usos y costumbres tradicionales en los estudiantes y los profesores universitarios. Se trata de un modelo formativo, tal y como se refleja en la Tabla 1 desde los preceptos de Lasnier (2000), donde el aprendizaje pasa a ser globalizado, constructivista y práctico; el estudiante se convierte en el protagonista de su propio proceso formativo, su propio entorno personal de aprendizaje (Khün, 2017); la enseñanza para a ser interactiva y cuyas actividades se diseñan en función de competencias; y la evaluación tiene un carácter más cualitativo e integral. 
Estudios Pedagógicos XLIV, $\mathrm{N}^{\circ}$ 2: 259-277, 2018

LAS COMPETENCIAS DOCENTES GENÉRICAS EN LOS GRADOS DE EDUCACIÓN. VISIÓN DEL PROFESORADO UNIVERSITARIO

Tabla 1. Elementos del nuevo modelo de formación basado en competencias

\begin{tabular}{|c|c|}
\hline Elementos & Formación por competencias \\
\hline Aprendizaje & $\begin{array}{l}\text { - Conocimientos aplicados: saber hacer } \\
\text { - Conocimientos globalizados } \\
\text { - Aprendizaje globalizado de conocimientos, destrezas y actitudes } \\
\text { - Influido por constructivismo } \\
\text { - Adquirido por actividades prácticas }\end{array}$ \\
\hline Estudiante & $\begin{array}{l}\text { - Ve más fácilmente los resultados a alcanzar (global) } \\
\text { - Motivación intrínseca } \\
\text { - Consignas generales para favorecer la iniciativa }\end{array}$ \\
\hline Enseñanza & $\begin{array}{l}\text { - Enseñanza interactiva } \\
\text { - Enfoque global } \\
\text { - Actividades en función de competencias }\end{array}$ \\
\hline Evaluación & $\begin{array}{l}\text { - Exigente } \\
\text { - Más bien subjetiva (juicio) } \\
\text { - Integra enseñanza, aprendizaje y evaluación } \\
\text { - Por tareas integradoras de aprendizaje } \\
\text { - Por comparación con los criterios de éxito: criterial } \\
\text { - Más bien cualitativa } \\
\text { - Integración de capacidades } \\
\text { - Fácil exactitud } \\
\text { - Sobre dominio de las competencias y estrategias de aprendizaje }\end{array}$ \\
\hline
\end{tabular}

Fuente. Lasnier (2000).

Este nuevo modelo formativo sobre el que se sustentan los diferentes planes de estudios se considera una preparación para la vida (Jiménez \& Bejarano, 2017, p. 41) y supone la creación de "un vínculo significativo entre la escuela, la Universidad y la vida". En esta dirección, pero desde un punto de vista productivo, el modelo competencial posibilita conectar los planes de estudios universitarios con las necesidades del contexto laboral (Julià, 2011).

Asimismo, el modelo va a condicionar la metodología que se lleve a cabo en el aula con el alumnado universitario y, por tanto, la posibilidad de desarrollar unas competencias en detrimento de otras, o, incluso, a mantenerse este nuevo elemento curricular en un segundo plano tras la primacía de los contenidos de aprendizaje (Ión \& Cano, 2012).

Uno de los motivos que conlleven a esta situación puede ser el alto grado de esfuerzo que han de realizar los docentes para planificar y desarrollar competencias en las diferentes materias que imparten. El estudio de Villa, Campo, Arranz, Villa y García (2013) apunta a que el profesorado universitario mostraba una postura positiva hacia el protagonismo del estudiante en este modelo competencial, pero la lección magistral, la falta de formación 
específica y la ausencia de coordinación entre el profesorado constituían igualmente una realidad. Sobre esta falta de formación específica en el modelo competencial también profundizan Villarroel y Bruna (2014), situación que se acrecienta cuando el profesorado se enfrenta por primera vez a la docencia universitaria (Perales, Sánchez \& Chiva, 2002).

De igual forma que la definición de competencia ha sido abordada por diferentes autorías, la clasificación o categorización de las competencias también ha sido analizada por diferentes autores y autoras (Bunk, 1994; Cano, 2005, 2007; Durán, Marcano \& Moronta, 2009; Gallego, 2000; Pazo \& Tejada, 2012; Tobón, 2006). Sin embargo, las más utilizadas en el ámbito universitario son las establecidas por la ANECA (2005) y por el Tuning Project (2007), que distinguen entre competencias genéricas y profesionales. Las primeras son de carácter básico, común y polivalente para el conjunto de los grados universitarios y las segundas "constituyen los saberes básicos, necesarios e imprescindibles para poder ejercer de forma segura y amplia una profesión concreta" (Salcines et al., en prensa).

El objetivo de esta investigación es, por tanto, determinar qué competencias genéricas se están implementando en las aulas universitarias y en qué grado se están desarrollando. A juicio de Yániz y Villardón (2006), este tipo de competencias deberían incluir habilidades cognitivas, metacognitivas, conocimientos instrumentales y actitudes. Por su parte, Martínez, Martínez y Muñoz (2008) proponen las siguientes áreas o dominios: comunicación, sentido ético, gestión de la información, profesionalismo, aprendizaje autónomo y desarrollo personal. En cuanto a los interrogantes de investigación, estos son dos: 1) ¿se fomenta el desarrollo de las competencias genéricas en las aulas universitarias?, 2) ¿existen diferencias estadísticamente significativas en el desarrollo de competencias genéricas en función de los datos que identifican al profesorado participante?

\section{MÉTODO}

El método seguido en esta investigación se fundamenta en una metodología cuantitativa de corte descriptivo y correlacional, que usa como técnica la encuesta y como instrumento el cuestionario (Arnal, Del Rincón \& Latorre, 1992).

En la primera parte del cuestionario se solicitan datos de identificación del profesorado universitario participante, en la segunda se pide su valoración sobre el grado de desarrollo de las competencias genéricas que ha de alcanzar el alumnado en las distintas asignaturas a partir de una escala de estimación con una gradación de 0 a 4 (nada, poco, algo, bastante y mucho). Este cuestionario, en su versión completa, incluye también la valoración de otro tipo de competencias.

Tras el diseño y validación del cuestionario, éste se aplicó en distintas universidades españolas, se depuraron los datos (Martínez-González, 2007) y se procedió a su tratamiento a través del SPSS (Universidad de Córdoba). Los estadísticos empleados han sido medidas de tendencia central y dispersión, $t$ de Student, ANOVA y la prueba post hoc (Scheffé) en función del tipo de variable seleccionada, entre otras.

La muestra participante estuvo constituida por 351 docentes universitarios, procedentes de 16 localidades españolas (Albacete, Barcelona, Córdoba, Huesca, León, Lérida, Madrid, Murcia, Salamanca, Santander, Segovia, Tenerife, Valencia, Valladolid, Vic y Vitoria) y 17 universidades diferentes. En las tablas 2, 3 y 4 se han recogido otras variables identificativas de los participantes. 
Estudios Pedagógicos XLIV, $\mathrm{N}^{\circ}$ 2: 259-277, 2018

LAS COMPETENCIAS DOCENTES GENÉRICAS EN LOS GRADOS DE EDUCACIÓN. VISIÓN DEL PROFESORADO UNIVERSITARIO

Tabla 2. Variables socio-demográficas de los participantes

\begin{tabular}{|c|c|c|}
\hline Variable independiente & Categorías & $\%$ \\
\hline \multirow{3}{*}{ Estudios en los que se imparte docencia } & Grado en Educación Primaria & 58.4 \\
\hline & Grado en Educación Infantil & 2.0 \\
\hline & CAFYD-INEF & 39.6 \\
\hline \multirow{6}{*}{ Tiempo de experiencia } & Menos de 5 años & 15.5 \\
\hline & Entre 6 y 10 años & 18.2 \\
\hline & Entre 11 y 15 años & 17.9 \\
\hline & Entre 16 y 20 años & 13.9 \\
\hline & Entre 21 y 25 años & 13.6 \\
\hline & Más de 25 años & 20.9 \\
\hline \multirow{5}{*}{ Edad } & Entre 26 y 30 años & 5.8 \\
\hline & Entre 31 y 40 años & 26.2 \\
\hline & Entre 41 y 50 años & 34.0 \\
\hline & Entre 51 y 60 años & 26.7 \\
\hline & Más de 60 años & 7.3 \\
\hline \multirow{2}{*}{ Título de doctor } & Sí & 75.4 \\
\hline & No & 24.6 \\
\hline \multirow{6}{*}{ Categoría profesional } & Catedrático de Universidad & 3.5 \\
\hline & Titular de Universidad (o CAEU) & 30.1 \\
\hline & Titular de Escuela Universitaria & 10.8 \\
\hline & Contratado doctor (tiempo completo) & 34.8 \\
\hline & Contratado a tiempo parcial o similar & 20.2 \\
\hline & Becario o similar & 0.6 \\
\hline \multirow{3}{*}{ Características asignaturas impartidas } & Teórica & 9.8 \\
\hline & Práctica & 8.9 \\
\hline & Teórico-práctica & 81.3 \\
\hline \multirow{5}{*}{ Carga lectiva semanal primer cuatrimestre } & Menos de 3 horas & 10.3 \\
\hline & Entre 4 y 6 horas & 27.0 \\
\hline & Entre 7 y 9 horas & 31.1 \\
\hline & Entre 10 y 12 horas & 15.5 \\
\hline & Más de 12 horas & 15.8 \\
\hline \multirow{5}{*}{ Carga lectiva semanal segundo cuatrimestre } & Menos de 3 horas & 17.0 \\
\hline & Entre 4 y 6 horas & 31.1 \\
\hline & Entre 7 y 9 horas & 24.6 \\
\hline & Entre 10 y 12 horas & 13.2 \\
\hline & Más de 12 horas & 14.1 \\
\hline
\end{tabular}


Tabla 3. Variables socio-demográficas de los participantes (continuación)

\begin{tabular}{|c|c|c|c|}
\hline Variable independiente & Categorías & & $\%$ \\
\hline \multirow{10}{*}{$\begin{array}{l}\text { Formación psicopedagógica } \\
\text { inicial }\end{array}$} & \multirow{2}{*}{ Estudios de Magisterio, Pedagogía, Psicología } & Sí & 48.0 \\
\hline & & No & 52.0 \\
\hline & \multirow{2}{*}{ Asignaturas aisladas en la carrera } & Sí & 40.2 \\
\hline & & No & 59.8 \\
\hline & \multirow{2}{*}{ Curso de Aptitudes Pedagógicas (CAP) } & Sí & 50.1 \\
\hline & & No & 49.9 \\
\hline & \multirow{2}{*}{ Formación universitaria complementaria } & Sí & 56.9 \\
\hline & & No & 43.1 \\
\hline & \multirow{2}{*}{ Otro tipo de formación } & Sí & 34.5 \\
\hline & & No & 65.5 \\
\hline \multirow{12}{*}{$\begin{array}{l}\text { Formación psicopedagógica } \\
\text { permanente }\end{array}$} & \multirow{2}{*}{$\begin{array}{l}\text { Cursos o talleres sobre aspectos docentes o } \\
\text { docencia universitaria }\end{array}$} & Sí & 82.3 \\
\hline & & No & 17.7 \\
\hline & \multirow{2}{*}{$\begin{array}{l}\text { Participación en proyectos de innovación } \\
\text { docente }\end{array}$} & Sí & 70.1 \\
\hline & & No & 29.9 \\
\hline & \multirow{2}{*}{ Formar parte de grupos de innovación docente } & Sí & 55.8 \\
\hline & & No & 44.2 \\
\hline & \multirow{2}{*}{$\begin{array}{l}\text { Asistencia a congresos de docencia } \\
\text { universitaria }\end{array}$} & Sí & 70.9 \\
\hline & & No & 29.1 \\
\hline & \multirow{2}{*}{$\begin{array}{l}\text { Escribir comunicaciones o artículos sobre } \\
\text { docencia universitaria }\end{array}$} & Sí & 62.5 \\
\hline & & No & 37.5 \\
\hline & \multirow{2}{*}{ Otros } & Sí & 29.6 \\
\hline & & No & 70.4 \\
\hline
\end{tabular}

Tabla 4. Variables socio-demográficas de los participantes (continuación)

\begin{tabular}{|l|c|c|}
\hline Variable independiente & Categorías & $\%$ \\
\hline \multirow{4}{*}{$\begin{array}{l}\text { Número de formas diferentes de haber realizado formación } \\
\text { psicopedagógica inicial }\end{array}$} & 0 formas & 4.0 \\
\cline { 2 - 3 } & 1 forma & 24.8 \\
\cline { 2 - 3 } & 2 formas & 28.5 \\
\cline { 2 - 3 } & 3 formas & 29.3 \\
\cline { 2 - 3 } & 4 formas & 10.0 \\
\cline { 2 - 3 } & 5 formas & 3.4 \\
\hline \multirow{4}{*}{$\begin{array}{l}\text { Número de formas diferentes de haber realizado formación } \\
\text { psicopedagógica permanente }\end{array}$} & 0 formas & 4.0 \\
\cline { 2 - 3 } & 1 forma & 13.1 \\
\cline { 2 - 3 } & 2 formas & 11.7 \\
\cline { 2 - 3 } & 3 formas & 14.2 \\
\cline { 2 - 3 } & 4 formas & 11.4 \\
\cline { 2 - 3 } & 5 formas & 30.2 \\
\hline
\end{tabular}


Por su parte, la media del número total de formas diferentes de haber realizado formación psicopedagógica inicial es de $2.26(d t=1.15)$, mientras que la media en formación permanente es de $3.68(d t=1.80)$.

\section{RESULTADOS}

Los resultados de esta investigación serán expuestos del siguiente modo: en primer lugar, el análisis descriptivo y discriminante de los ítems que componen el cuestionario; en segundo lugar, se ofrecerá el estudio psicométrico del cuestionario y, por último, se planteará un análisis inferencial para determinar el cumplimiento o no de los interrogantes de investigación.

\subsection{ANÁLISIS DESCRIPTIVO Y DISCRIMINANTE DE LOS ÍTEMS}

Para conocer el comportamiento de los ítems, se ha analizado la media, la desviación típica y el Alfa de Cronbach si se eliminaba el ítem de cada una de las preguntas del cuestionario. Las medias oscilaron entre 1.27 y 2.58, siendo la media de la escala de 2. De los 12 ítems, 3 se encontraron por debajo de esta media. Por su parte, la desviación típica informa de la variabilidad de las respuestas en todos los casos, salvo en un ítem. El Alfa de Cronbach del cuestionario es de .907, en la Tabla 5 se puede observar que solo mejoraría el mismo si se eliminase el ítem 10.

Tabla 5. Análisis de los ítems

\begin{tabular}{|l|c|c|c|c|}
\hline \multicolumn{1}{|c|}{ Ítems } & $\mathrm{N}$ & $\bar{X}$ & $\mathrm{DT}$ & $\begin{array}{c}\alpha \text { si se } \\
\text { elimina }\end{array}$ \\
\hline $\begin{array}{l}\text { 1. Conocer características de la organización de los centros } \\
\text { educativos }\end{array}$ & 328 & 1,54 & 1,334 &, 900 \\
\hline 2. Elaborar propuestas de cambio de la realidad educativa & 328 & 2,29 & 1,303 &, 899 \\
\hline 3. Organizar y animar situaciones de aprendizaje & 328 & 2,59 & 1,031 &, 901 \\
\hline 4. Gestionar la progresión de los aprendizajes & 328 & 2,48 &, 942 &, 901 \\
\hline $\begin{array}{l}\text { 5. Elaborar y poner en práctica estrategias de atención a la } \\
\text { diversidad }\end{array}$ & 328 & 2,07 & 1,203 &, 897 \\
\hline $\begin{array}{l}\text { 6. Implicar al alumnado en su aprendizaje y en la vida del } \\
\text { centro }\end{array}$ & 328 & 2,16 & 1,185 &, 895 \\
\hline 7. Trabajar en equipo con otros docentes & 328 & 2,28 & 1,245 &, 897 \\
\hline 8. Participar en la gestión del centro & 328 & 1,27 & 1,160 &, 895 \\
\hline 9. Informar e implicar a las familias & 328 & 1,34 & 1,297 &, 898 \\
\hline $\begin{array}{l}\text { 10. Utilizar las tecnologías de la información y la } \\
\text { comunicación }\end{array}$ & 328 & 2,58 & 1,025 &, 908 \\
\hline 11. Afrontar los deberes y los dilemas éticos de la profesión & 328 & 2,34 & 1,216 &, 898 \\
\hline 12. Organizar la propia formación continua & 328 & 2,10 & 1,133 &, 900 \\
\hline
\end{tabular}


Según García, Gil y Rodríguez (1995), es necesario que los ítems cuenten con un poder de discriminación elevado, ya que así refuerzan el carácter unidimensional de la herramienta. Para saber si los ítems de la escala lo tenían, la suma total se recodificó en tres grupos:

1 = Grupo Bajo (valor mínimo, percentil 33): (1,18.83)

$2=$ Grupo Medio (percentil 34, percentil 66): (18.84, 27.27)

3 = Grupo Alto (percentil 67, valor máximo): (27.28, 43.33)

Una vez configurados los grupos, se aplicó una prueba de $t$ para muestras independientes que posibilitó el establecimiento de diferencia estadística (n.s.=.05) entre los grupos que puntuaron bajo y alto en los distintos ítems. En este sentido, el 100\% de los ítems que componen la escala posee un alto poder de discriminación, ya que obtuvo $(\mathrm{p}=.000)$.

\subsection{ESTUDIO PSICOMÉTRICO DEL CUESTIONARIO}

Como ya se ha comentado, el análisis de consistencia interna de la escala arrojó un Alfa de Cronbach en el conjunto de la escala de .907.

Para realizar el estudio psicométrico del cuestionario se halló también la medida de adecuación muestral KMO para las 12 variables de la escala. Los resultados obtenidos (.907) evidenciaron garantías de conseguir un buen resultado en el Análisis Factorial. Asimismo, la prueba de esfericidad de Bartlett $(1950,265 ; \mathrm{p}=.000)$ indicaba la adecuación del Análisis Factorial para la explicación de los datos. El análisis de la matriz de correlaciones (determinante=.002) también sugería su empleo. El Análisis Factorial se inició con la obtención de comunalidades a través del análisis de componentes principales para toda la escala, solo dos de ellas fueron inferiores a .5. En el caso concreto de las competencias analizadas oscilaron entre .511 y .775 .

El resultado fue la configuración de dos factores, pero en la matriz de estructura factorial se podía apreciar que todas las variables saturaban en el primer factor. La varianza explicada por estos dos factores fue de $59.001 \%$, lo que la situaría por encima del límite establecido por Henson y Roberts (2006). El primero de los factores explicaba un $31.592 \%$ de la varianza, mientras que el segundo lo hacía con un $27.410 \%$.

Todas las variables obtuvieron unas cargas comprendidas entre .485 y .789 . Posteriormente, se realizó una rotación de los factores (normalización Varimax con Kaiser) para comprobar si se producía alguna modificación, hecho que tuvo lugar tal y como se aprecia en la Tabla 6.

La existencia de un factor predominante, aunque no prueba, sí apoyaba en un principio la interpretación de unidimensionalidad del constructo medido por la escala. Este carácter unidimensional de carácter empírico se complementaba con una unidimensionalidad conceptual. Sin embargo, al realizar la rotación, la unidimensionalidad de la escala quedaba comprometida. 
Tabla 6. Matriz de componentes

\begin{tabular}{|l|c|c|c|c|}
\hline \multirow{2}{*}{\multicolumn{1}{|c|}{ Competencias }} & \multicolumn{2}{|c|}{$\begin{array}{c}\text { Factores } \\
\text { sin rotar }\end{array}$} & \multicolumn{2}{c|}{$\begin{array}{c}\text { Factores } \\
\text { con rotación }\end{array}$} \\
\cline { 2 - 6 } & 1 & 2 & 1 & 2 \\
\hline 1. Conocer características de la organización de los centros educativos &, 693 &,- 369 &, 762 &, 190 \\
\hline 2. Elaborar propuestas de cambio de la realidad educativa &, 715 &, 002 &, 530 &, 480 \\
\hline 3. Organizar y animar situaciones de aprendizaje &, 664 &, 532 &, 137 &, 840 \\
\hline 4. Gestionar la progresión de los aprendizajes &, 670 &, 513 &, 154 &, 830 \\
\hline 5. Elaborar y poner en práctica estrategias de atención a la diversidad &, 746 &, 184 &, 430 &, 636 \\
\hline 6. Implicar al alumnado en su aprendizaje y en la vida del centro &, 789 &,- 065 &, 630 &, 480 \\
\hline 7. Trabajar en equipo con otros docentes &, 747 &,- 023 &, 570 &, 483 \\
\hline 8. Participar en la gestión del centro &, 782 &,- 405 &, 851 &, 223 \\
\hline 9. Informar e implicar a las familias &, 726 &,- 430 &, 827 &, 167 \\
\hline 10. Utilizar las tecnologías de la información y la comunicación &, 485 &, 023 &, 345 &, 342 \\
\hline 11. Afrontar los deberes y los dilemas éticos de la profesión &, 721 &,- 010 &, 542 &, 476 \\
\hline 12. Organizar la propia formación continua &, 686 &, 165 &, 399 &, 582 \\
\hline
\end{tabular}

\subsection{RESULTADOS DESCRIPTIVOS E INFERENCIALES}

En este apartado se muestran los resultados hallados en esta investigación tras aplicar diferentes pruebas estadísticas ( $t$ de Student y ANOVA). En general, las medias obtenidas en la valoración del profesorado sobre el desarrollo de competencias genéricas en sus aulas se sitúan por encima de la media de la escala en la mayoría de los casos, tal y como se podía apreciar en la Tabla 5. En este sentido, el desarrollo de competencias como "Conocer características de la organización de los centros educativos", "Participar en la gestión del centro" e "Informar e implicar a las familias" son las menos movilizadas entre el profesorado. Por el contrario, las que más se favorecen en las aulas son "Organizar y animar situaciones de aprendizaje" y "Utilizar las tecnologías de la información y la comunicación".

La edad no constituye un condicionante para el desarrollo de competencias genéricas en las diferentes materias, al igual que la carga lectiva docente, tanto en el primer como en el segundo cuatrimestre, o el número de modalidades de formación inicial del docente. No obstante, la localidad de procedencia del docente, su categoría profesional, su experiencia docente, los estudios y el tipo de asignatura que imparte, así como el número de modalidades realizadas en la formación permanente condicionan en mayor o menor medida la movilización de competencias genéricas en el aula (ver Tabla 7).

En el caso de la localidad, se identificaron diferencias estadísticas significativas, pero al aplicar la prueba de Scheffé para determinar entre qué provincias se establecían dichas diferencias, los resultados fueron negativos. 
En cuanto a los estudios en los que el profesorado imparte docencia, cabe destacar que el desarrollo de las competencias genéricas es mayor en el Grado de Educación Primaria que en los estudios de CAFYD-INEF. Por su parte, el tiempo de experiencia también resulta significativo, ya que a menos experiencia docente, mayor desarrollo de dichas competencias, situándose la franja de menor implicación en la comprendida entre los 21 y los 25 años de trabajo.

En lo que concierne a la categoría profesional, el profesorado con menor estabilidad laboral, por tener un puesto a tiempo parcial, es el que más moviliza las competencias genéricas en el aula, frente al profesorado titular de Universidad.

En el caso de la tipología de clase que se imparte, es el profesorado que tiene asignaturas teórico-prácticas el que más favorece el desarrollo de las competencias genéricas en el aula frente al que tiene asignaturas teóricas.

Respecto al número de modalidades de formación permanente en las que ha participado el profesorado, se puede afirmar que a mayor número de modalidades $(5$ o 6 ), más movilización de las competencias genéricas.

El desarrollo de competencias genéricas en las aulas universitarias se vincula también, sobre todo, con la realización de estudios psicopedagógicos por parte del profesorado y con la formación permanente del mismo, tal y como se puede observar en la Tabla 8. Asimismo, no se encontraron diferencias estadísticamente significativas entre este desarrollo competencial y las variables sexo, posesión del título de doctor, formación inicial a través de asignaturas aisladas a lo largo de la carrera, a través de la realización del CAP, a través de cursos ofertados por la Universidad u otro tipo de formación.

Tabla 7. Resultados estadísticos tras aplicar la prueba de ANOVA

\begin{tabular}{|c|c|c|c|c|c|}
\hline Variable independiente & $\mathrm{F}$ & $\mathrm{p}$ & $\begin{array}{l}\text { Scheffé } \\
(\mathrm{p})\end{array}$ & $\begin{array}{c}\text { Diferencias } \\
\text { de medias } \\
(\mathrm{I}-\mathrm{J})\end{array}$ & $\bar{X}$ \\
\hline Localidad & 4.688 & .000 & - & - & - \\
\hline $\begin{array}{l}\text { Estudios en los que imparte } \\
\text { docencia }\end{array}$ & 17.352 & .000 & .000 & -.517 & $\begin{array}{l}\text { G. Primaria= } 2.22 \\
\text { CAFYD-INEF= } 1.70\end{array}$ \\
\hline Tiempo de experiencia & 5.395 & .000 & $\begin{array}{l}.015 \\
.032 \\
.008\end{array}$ & $\begin{array}{l}.637 \\
.571 \\
.690\end{array}$ & $\begin{array}{l}<5 \text { años }=2.21 \text { y entre } 21 \text { y } \\
25 \text { años }=1.57 \\
\text { Entre } 6 \text { y } 10 \text { años }=2.14 \text { y } \\
\text { entre } 21 \text { y } 25 \text { años }=1.57 \\
\text { Entre } 16 \text { y } 20 \text { años }=2.26 \text { y } \\
\text { entre } 21 \text { y } 25 \text { años }=1.57\end{array}$ \\
\hline Categoría profesional & 4.300 & .001 & .010 & -.495 & $\begin{array}{l}\text { Titular }=1.77 \\
\text { Tiempo parcial }=2.27\end{array}$ \\
\hline Tipo de clases que imparte & 6.157 & .002 & .005 & -.520 & $\begin{array}{l}\text { Teóricas }=1.55 \\
\text { Teórico-prácticas }=2.07\end{array}$ \\
\hline $\begin{array}{l}\text { Número de modalidades de } \\
\text { formación permanente }\end{array}$ & 7.105 & .000 & $\begin{array}{l}.014 \\
.016 \\
.001 \\
.003\end{array}$ & $\begin{array}{l}-.893 \\
-.926 \\
-.689 \\
-.722\end{array}$ & $\begin{array}{l}0=1.36 \text { y } 5=2.25 \\
0=1.36 \text { y } 6=2.28 \\
1=1.56 \text { y } 5=2.25 \\
1=1.56 \text { y } 6=2.28\end{array}$ \\
\hline
\end{tabular}


Estudios Pedagógicos XLIV, $\mathrm{N}^{\circ}$ 2: 259-277, 2018

LAS COMPETENCIAS DOCENTES GENÉRICAS EN LOS GRADOS DE EDUCACIÓN. VISIÓN DEL PROFESORADO UNIVERSITARIO

Tabla 8. Resultados estadísticos tras aplicar la prueba de t de Student

\begin{tabular}{|lccl|}
\hline \multicolumn{1}{|c}{ Variable independiente } & $\mathrm{t}$ & $\mathrm{p}$ & \multicolumn{1}{c|}{$\bar{X}$} \\
\hline F.I. estudios de Magisterio, Pedagogía, Psicología & 4.696 & .000 & $\begin{array}{l}\text { Sí=2.22 } \\
\text { No=1.81 }\end{array}$ \\
\hline F.P. cursos o talleres sobre docencia universitaria & 2.139 & .033 & $\begin{array}{l}\text { Sí }=2.04 \\
\text { No=1.79 }\end{array}$ \\
\hline F.P. participación en proyectos de innovación & 3.913 & .000 & $\begin{array}{l}\text { Sí }=2.11 \\
\text { No=1.74 }\end{array}$ \\
\hline F.P. formar parte de grupos de innovación docente & 5.852 & .000 & $\begin{array}{l}\text { Sí }=2.23 \\
\text { No=1.72 }\end{array}$ \\
\hline F.P. asistencia a congresos de docencia universitaria & 4.955 & .000 & $\begin{array}{l}\text { Sí }=2.14 \\
\text { No=1.66 }\end{array}$ \\
\hline F.P. escribir comunicaciones o artículos sobre docencia universitaria & 5.338 & .000 & $\begin{array}{l}\text { Sí=2.20 } \\
\text { No=1.71 }\end{array}$ \\
\hline
\end{tabular}

\section{DISCUSIÓN DE LOS RESULTADOS Y CONCLUSIONES}

La discusión de los resultados expuestos en el apartado anterior partirá del objetivo propuesto en la investigación y continuará con la respuesta a los interrogantes planteados. En este sentido, sobre el objetivo "determinar qué competencias genéricas se están implementando en las aulas universitarias y en qué grado se están desarrollando", se puede mencionar que las más trabajadas en las aulas se vinculan a la elaboración de propuestas de cambio de la realidad educativa, la organización y animación de situaciones de aprendizaje y la gestión de la progresión de los aprendizajes, todas ellas propias del ámbito de la planificación curricular y facilitadoras del aprendizaje para poder movilizar las competencias clave en los centros de enseñanza básica (educación primaria y educación secundaria obligatoria) (De-Juanas, Martín \& Pesquero, 2016).

Junto a ellas, la elaboración y puesta en práctica de estrategias de atención a la diversidad es otra de las competencias más desarrolladas en el contexto universitario, quizá debido a que la diversidad resulta un factor inherente al sistema educativo actual y así se ha puesto de manifiesto en los diseños curriculares de formación inicial del profesorado. Sin embargo, como señalan Figueredo y Ortiz (2017), no todos los factores de diversidad son tratados del mismo modo en los planes de estudio. En este sentido, estos autores han demostrado escasas referencias a la diversidad cultural en los diseños curriculares andaluces. Habría, por tanto, que profundizar sobre qué aspectos o factores de diversidad se está incidiendo en las aulas universitarias.

Mientras que el profesorado participante en esta investigación considera que la competencia "implicación del alumnado en su aprendizaje y en la vida del centro" se trabaja en el aula, la perspectiva del alumnado en otros estudios es contradictoria, al considerar que no poseen la habilidad de regular su propio aprendizaje (Rodríguez \& Revilla, 2016). Por tanto, habría que contrastar la opinión de los docentes con las de sus propios alumnos para comprobar estos extremos.

Otra de las competencias que más se fomentan es el trabajo en equipo con otros docentes. Diferentes proyectos de innovación docente están propiciando que el profesorado uni- 
versitario ponga en marcha nuevas metodologías docentes como el aprendizaje-servicio para desarrollar habilidades de trabajo en equipo (Cámara \& Pegalajar, 2017). Al mismo tiempo, estudios como los desarrollados por Jayasingam, Fujiwara \& Thurasamy (2016) y Lluch, Fernández-Ferrer, Pons y Cano (2017) muestran que los empleadores valoran como muy positiva la competencia de trabajo en equipo, pero Cabrera, Portillo y Prades (2016, p. 102) en sus investigaciones aportan que los empleadores suelen vincular esta competencia con una "actitud positiva y de colaboración, más que de trabajo en equipo propiamente dicho".

Por su parte, la utilización de las tecnologías de la información y la comunicación es otra de las competencias con mayor movilización en el aula universitaria (Almarghani \& Mijatovic, 2017; Engel, Heinz \& Sonntag, 2017). No obstante, aunque el alumnado emplee estas tecnologías, desconocemos si generan el perfil propio de un nativo digital capaz de producir, difundir y consumir cultura a través de Internet, carencias que han evidenciado investigaciones como la realizada por Castellanos, Sánchez y Calderero (2017) con el alumnado del Grado de Maestro de Educación Primaria. En esta misma dirección, Khün (2017) apunta que el alumnado universitario no se encuentra preparado para diseñar o rediseñar su propio Personal Learning Environment (PLE), ya que se resiste a emplear herramientas basadas en la red para aprender debido a que no quieren poner en riesgo sus calificaciones. En este sentido, a pesar de que el profesorado participante valora el trabajo que se lleva a cabo en el aula para que el alumnado adquiera la competencia de organización de la propia formación continua, trabajos como el de Khün (2017) mostrarían las dificultades del alumnado para desarrollar su propio entorno de aprendizaje, tanto en el contexto universitario como tras su salida del mismo.

El afrontamiento de los deberes y los dilemas éticos de la profesión es considerado también por el profesorado participante como una competencia trabajada en el aula. No obstante, hay autores que apuntan la dificultad de que esta se aborde de manera integral en el contexto universitario, planteándose como un reto que hay que alcanzar, sobre todo, en un momento de crisis de valores como el que se vive en la actualidad (Martino \& Naval, 2013; Scriven, 2016).

Por otro lado, aquellas competencias que se relacionan con la gestión de un centro educativo y la relación con el contexto socio-familiar son las menos movilizadas en el aula, posiblemente debido a un mayor desconocimiento de estos aspectos por parte del profesorado universitario, ya que son competencias que requieren un conocimiento exhaustivo y específico de los centros educativos y de la necesidad que expresaban Jiménez y Bejarano (2017, p. 41) de crear "un vínculo significativo entre la escuela, la Universidad y la vida".

En cuanto al grado en el que las competencias genéricas son desarrolladas en el aula, si bien la mayoría de ellas se sitúan por encima de la media de la escala, esto resulta, a nuestro juicio, insuficiente, sobre todo cuando los procesos de acomodación al Espacio Europeo de Educación Superior ya se han producido y nos encontramos en los primeros años de su consolidación, lo que nos lleva a pensar si los contenidos curriculares siguen siendo los que marcan la metodología docente universitaria (Ión \& Cabo, 2012; Villa et al., 2013). En esta línea, el trabajo de Suárez (2017) evidencia un descontento por parte del alumnado del Grado de Psicología en cuanto al nivel competencial alcanzado, situación que también es constatada por Kamphorst, Hofman, Jansen \& Terlouw (2012).

En lo que respecta a los interrogantes de investigación, el primero de ellos aludía a si se fomenta el desarrollo de las competencias genéricas en las aulas universitarias. En este 
sentido, y vinculado al objetivo planteado en la investigación, hemos de afirmar que este fomento tiene lugar, pero que es escaso e irregular y que depende de cada competencia. El segundo hacía referencia a si existían diferencias estadísticamente significativas en el desarrollo de competencias genéricas en función de los datos que identifican al profesorado participante. Efectivamente, existen diferencias entre los encuestados. Así, se puede señalar que aspectos como la formación previa, la experiencia docente, la categoría profesional, los estudios y el tipo de asignatura en el que se ejerce la docencia resultan factores condicionantes de un mayor o menor desarrollo de las competencias genéricas en las aulas universitarias. Sin embargo, el aspecto más relevante de este condicionamiento se encuentra en la formación permanente del profesorado, que pone de manifiesto la necesidad de que la Universidad española siga apostando e invirtiendo en las distintas modalidades de formación permanente para sus docentes con el fin de cumplir con el objetivo europeo de un sistema formativo de carácter competencial.

\section{REFERENCIAS BIBLIOGRÁFICAS}

Almarghani, E.M., \& Mijatovic, I. (2017). Factors affecting student engagement in HEIs - it is all about good teaching. Teaching in Higher Education, 22(8), 940-956. doi:10.1080/13562517.2 017.1319808

ANECA-Agencia Nacional de Evaluación de la Calidad y Acreditación. (2005). Libro blanco título de Grado en Magisterio, 1. Madrid: Sirius. Recuperado de: http://www.aneca.es/var/ media/150404/libroblanco_jun05_magisterio1.pdf.

Ángulo J.F. (2009). La voluntad de distracción: Las competencias en la universidad. En J. Gimeno Sacristán (Coord.), Educar por competencias, ¿qué hay de nuevo? (pp. 176-205). Madrid: Morata.

Arnal, J., Del Rincón, D., \& Latorre, A. (1992). Investigación educativa. Fundamentos y metodología. Barcelona: Labor.

Barnett, R. (2001). Los límites de las competencias. Barcelona: Gedisa.

Bloom, B.S. (1956). Taxonomy of educational objectives; the classification of educational goals. New York: Longmans, Green.

Bronfenbrenner, U. (1987). La ecología del desarrollo humano. Barcelona: Paidós.

Bunk, G.P. (1994). La transmisión de las competencias en la formación y perfeccionamiento de profesionales en la RFA. Revista Europea de Formación Profesional, 1, 8-14.

Cabrera, N., Portillo, M.C., \& Prades, A. (2016). Las competencias de los graduados universitarios y su evaluación. La perspectiva de los empleadores. En E. Cano y M. Fernández (Eds.), Evaluación por competencias: la perspectiva de las primeras promociones de graduados en el EEES (pp. 95-112). Barcelona: Octaedro.

Cámara, A.M., \& Pegalajar, M.C. (2017). Valoración del alumnado del grado de educación primara acerca de la metodología de aprendizaje-servicio. Revista Electrónica de Investigación y Docencia, 18, 7-24. doi:10.17561/reid.v0i18.3124

Cano, E. (2005). Cómo mejorar las competencias de los docentes: guía para la autoevaluación y desarrollo de las competencias del profesorado. Barcelona: Graó.

Cano, E. (2007). Las competencias de los docentes. En Ministerio de Educación y Ciencia (Ed.), El desarrollo de competencias docentes en la formación del profesorado (pp. 33-60). Madrid: Secretaría General Técnica.

Cano, E. (Ed.). (2012). Aprobar o aprender. Estrategias de evaluación en la sociedad red. Barcelona: Laboratori de MitjansInteractius, Universitat de Barcelona. 
Cascante, C. (2004). La reforma de los Planes de Estudio. Un análisis político de los discursos sobre la Formación Inicial de los Profesionales de la Educación. Revista Interuniversitaria de Formación del Profesorado, 18(3), 145-167.

Castellanos, A., Sánchez, C., \& Calderero, J.F. (2017). Nuevos modelos tecnopedagógicos. Competencia digital de los alumnos universitarios. Revista Electrónica de Investigación Educativa, 19(1), 4-9. doi:10.24320/redie.2017.19.1.1148

Clemente, M. (2010). Diseñar el currículum. Prever y representar la acción. En J. Gimeno Sacristán (Coord.), Saberes e incertidumbres del currículum (pp. 269-293). Madrid: Morata.

Coll, C. (2007). Las competencias en la educación escolar: algo más que una moda y mucho menos que un remedio. Aula de Innovación Educativa, 161, 34-39.

Declaración de Bolonia. (1999). El Espacio Europeo de Educación Superior. Declaración conjunta de los ministros europeos de educación reunidos en Bolonia el 19 de junio de 1999. Recuperado de: http://tecnologiaedu.us.es/mec2011/htm/mas/2/21/6.pdf.

De-Juanas, A., Martín, R., \& Pesquero, E. (2016). Teaching competences necessary for developing key competences of primary education students in Spain: teacher assessment. Teacher Development, 20, 123-145. doi:10.1080/13664530.2015.1101390

Delamare Le Deist, F., \& Winterton, J. (2005). What is competence? Human Resource Development International, 8(1), 27-46.

Durán, J.A., Marcano, N., \& Moronta, M. (2009). Competencias investigativas del docente de educación básica. Revista Laurus, 15(39), 138-165. Recuperado de http://www.redalyc.org/src/ inicio/ArtPdfRed.jsp?iCve=76120651007

Engel, M., Heinz, M. \& Sonntag, R. (2017). Flexibilizing and Customizing Education using Inverted Classroom Model. Information Systems Management, 34(4), 378-389.

Estévez, E. (2002). Enseñar a aprender. México: Trillas.

Fernández-March, A. (2005). Taller sobre el proceso de aprendizaje-enseñanza de competencias. Recuperado de http://www.uv.es/adedch/documentos/Taller.pdf.

Figueredo, V., \& Ortiz, L. (2017). Profesorado y diversidad cultural: Análisis de la formación inicial del profesorado en interculturalidad. International Journal of Educational Research and Innovation, 9, 46-71.

Gallego, M. (2000). Gestión humana basada en competencias. Contribución efectiva al logro de los objetivos organizacionales. Revista Universidad EAFIT, 119, 63-71.

García, E., Gil, J., \& Rodríguez, G. (1995). Introducción a la teoría clásica de los test. Sevilla: Grupo Delta.

González, J., \& Wagenaar, R. (2003). Tuning Educational Structures in Europe. Bilbao: Universidad de Deusto.

Gros, B. y Bernat, A. (2008). El aprendizaje de competencias de alfabetización digital a través de los videojuegos. Aula de Innovación Educativa, 176, 12-16.

Henson, R.K., \& Roberts, J.K. (2006). Use of exploratory Factor Analysis in published research: Common errors and some comment on improved practice. Educational and Psychological Measurement, 66, 393-416.

Ion, G., \& Cano, E. (2012). La formación del profesorado universitario para la implementación de la evaluación por competencias. Educación XX1, 5(2), 249-270. doi:10.5944/educxx1.15.2.141

Jayasingam, S., Fujiwara, Y., \& Thurasamy, R. (2016). I am competent so I can be choosy': choosiness and its implication on graduate employability. Studies in Higher Education, 2, 1-16. doi:10.108 0/03075079.2016.1221918

Jiménez, A., \& Bejarano, C.A. (2017). ¿Qué entendemos por competencia? Una perspectiva desde las definiciones. En A. Jiménez (Coord.), Competencias educativas e innovación (pp. 17-49). Salamanca: Universidad Pontificia de Salamanca, Servicio de Publicaciones.

Juliá, M.T. (2011). Formación basada en competencias: Aportes a la calidad de los aprendizajes en la formación de psicólogos. En J. Catán (Ed.), Psicología educacional. Proponiendo rumbos, 
Estudios Pedagógicos XLIV, N $^{\circ}$ 2: 259-277, 2018

LAS COMPETENCIAS DOCENTES GENÉRICAS EN LOS GRADOS DE EDUCACIÓN. VISIÓN DEL PROFESORADO UNIVERSITARIO

problemáticas y aportaciones (pp. 245-269). La Serena: Editorial Universidad de la Serena.

Kamphorst, J.C., Hofman, W.H.A., Jansen, E.P.W.A., \& Terlouw, C. (2012). The relationship between perceived competence and earned credits in competence-based higher education. Assessment \& Evaluation in higher Education, 38(6), 646-661. doi:10.1080/02602938.2012.680015

Krathwohl, D.R., Bloom, B.S., \& Masia, B.B. (1964). Taxonomy of educational objectives: Handbook II: Affective domain. New York: David McKay Co.

Khün, C. (2017). Are Students Ready to (re)-Design their Personal Learning Environment? The case of the E-Dynamic Space. Journal of New Approaches in Educational Research, 6(1), 11-19.

Lasnier, F. (2000). Réussir la formation par compétences. Montreal: Guérin.

Lavega P. (2008). Educación física y mercado laboral. Competencias profesionales. Cultura, Ciencia y Deporte, 8(3), 123-131.

Le Boterf, G. (2001). Ingeniería de las competencias. Barcelona: Epise, Training Club y Ediciones Gestión 2000.

Lozano, R., Merrill, M.Y., Sammalisto, K., Ceulemans, K., \& Lozano, F.J. (2017). Connecting Competences and Pedagogical Approaches for Sustainable Development in Higher Education: A Literature Review and Framework Proposal. Sustainability, 9, 1-15. doi:10.3390/su9101889

Lluch, L., Fernández-Ferrer, M., Pons, L., \& Cano, E. (2017). Competencias profesionales de los egresados universitarios: estudios de casos en cuatro titulaciones. Revista Qurriculum, 30, 49-64. Recuperado de https://riull.ull.es/xmlui/bitstream/handle/915/6135/Q_30_\%282017\%29_03. pdf? sequence $=1 \&$ is Allowed $=\mathrm{y}$

Martín囚Domínguez, J., \& Lavega, P. (2013). Enseñar competencias en el Espacio Europeo de Educación Superior. Revista Electrónica Interuniversitaria de Formación del Profesorado, 16(1), 1『4. doi:10.6018/reifop.16.1.179391

Martínez, P., Martínez, M., \& Muñoz, J.M. (2008). Formación basada en competencias en educación sanitaria: aproximaciones a enfoques y modelos de competencia. RELIEVE, 14(2), 1-23. Recuperado de http://www.uv.es/RELIEVE/v14n2/RELIEVEv14n2_1.htm

Martínez-González, R.A. (2007). La investigación en la práctica educativa: guía metodológica de investigación para el diagnóstico y evaluación en los centros docentes. Madrid: CIDE/MEC.

Martino, S., \& Naval, C. (2013). La formación ética y cívica en la Universidad. El papel de los docentes. Edetania, 43, 161-186. Recuperado de https://dialnet.unirioja.es/descarga/articulo/4406440.pdf.

Monereo, C., \& Pozo, J.I. (2007). Competencias para (con)vivir con el siglo XXI. Cuadernos de Pedagogía, 370, 12-18.

OCDE-DESECO. (2002). Definition and Selection of Competencies: Theoretical and Conceptual Foundations. Recuperado de http://www.portal-stat.admin.ch/deseco/index.htm.

Pazo, C.I., \& Tejada, J. (2012). Las competencias profesionales en Educación Física, Retos. Nuevas tendencias en Educación Física, Deporte y Recreación, 22, 5-8. Recuperado de http://retos.org/ pdf/retos22.pdf.

Perales, M. J., Sánchez, P., \& Chiva, I. (2002). El "Curso de Iniciación a la Docencia Universitaria" como experiencia de formación de profesores universitarios noveles en la Universitat de València. Un sistema de evaluación. Revista Electrónica de Investigación y Evaluación Educativa, 8(1). Recuperado de http://www.uv.es/RELIEVE/v8n1/RELIEVEv8n1_4.htm.

Perrenoud, P. (2004). Diez nuevas competencias para enseñar: invitación al viaje. Barcelona: Graó.

Ramírez-García, A. (2013). El desarrollo de las competencias básicas en educación primaria y secundaria a través del videojuego. En V. Marín Díaz (Coord.), Los videojuegos y juegos digitales como materiales educativos (pp. 61-78). Madrid: Síntesis.

Ramos, S. (2005). El desarrollo de las competencias didácticas: un reto en la formación de los futuros docentes de primaria. Educar, 35, 51-76.

Rodríguez-Diéguez, J.L. (1980). Didáctica General. Madrid: Cincel.

Rodríguez, M., \& Revilla, P. (2016). Las competencias generales y transversales del Gradode Logopedia desde la perspectiva del alumno. Educatio Siglo XXI, 34(1), 113-136. doi:10.6018/j/253241. 
Román, J.M. (2007). Adaptaciones pedagógicas exigidas por las "competencias clave" de los títulos del EEES. En I. Rodríguez Escanciano (Ed.), Inserción laboral y EEES: aplicación a las ciencias de la información (pp. 27-44). Valladolid: Servicio Publicaciones de la UEMC.

Rué, J. (2004). Conceptualizar el aprendizaje y la docencia en la Universidad mediante los ECTS. Revista Interuniversitaria de Formación del Profesorado, 18(3), 179-195.

Salcines, González, Ramírez., \& Martínez. (en prensa). Validación de la Escala de Autopercepción de Competencias Transversales y Profesionales de Estudiantes de Educación Superior. Profesorado. Revista de Currículum y Formación del Profesorado.

Scriven, M. (2016). Professorial Ethics. The Jounal of Higher Education, 53(3), 307-317. doi:10.10 80/00221546.1982.11780456

Sierra, B., Méndez-Giménez, A., \& Mañana-Rodríguez, J. (2007). La programación por competencias básicas: hacia un cambio metodológico interdisciplinar. Revista Complutense de Educación, 24(1), 165-184.

Suárez, X.A. (2017). Percepción de adquisición de competencias genéricas en estudiantes de Psicología que inician y finalizan su formación. Actualidades investigativas en Educación, 17(3), 1-21. doi:10.15517/aie.v17i3.30277.

Tejada, J. (2009). Profesionalización docente en el escenario de la Europa de 2010. Una mirada desde la formación. Revista de Educación, 349, 463-477.

Tobón, S. (2006). Formación basada en competencias. Pensamiento complejo, diseño curricular y didáctica. Bogotá: Ecoe.

Tuning Project. (2007). Educational Structures in Europe. Competences. University of Groninge. University of Deusto. Recuperado de http://www.unideusto.org/tuningeu/competences.html.

Villa, A., Campo, L., Arranz, S., Villa, O., \& García, A. (2013). Valoración del profesorado de Magisterio sobre el aprendizaje basado en competencias implantado. Profesorado. Revista de currículum y formación del profesorado, 17(3), 35-55. Recuperado de http://recyt.fecyt.es/ index.php/profesorado/article/view/41981/23964.

Villarroel, V., \& Bruna, D. (2014). Reflexiones en torno a las competencias genéricas en educación superior: Un desafío pendiente. Revista Psicoperspectivas, 13(1), 23-24. doi:10.5027/ PSICOPERSPECTIVAS-VOL13SSUE1-FULLTEXT-335

Yáñiz, C., \& Villardón, L. (2006). Planificar competencias para promover el aprendizaje. El reto de la sociedad del conocimiento para el profesorado universitario. Bilbao: Cuadernos Monográficos del ICE, 12.

Zabala, A., \& Arnau, L. (2008). 11 ideas clave. Cómo aprender y enseñar competencias. Barcelona: Graó.

Zabalza, M. A. (2002). Diseño curricular en la universidad. Competencias del docente universitario. Madrid: Narcea. 
